\title{
LAS INTERACCIONES ECOLÓGICAS Y SU RELACIÓN CON LA CONSERVACION DE LA BIODIVERSIDAD
}

\section{Victor Rico-Gray}

Departamento de ECOlOgÍA ApliCADA

Instituto de Ecología, A.C., Xalapa, Veracruz, MéXico

\section{INTRODUCCIÓN}

La biodiversidad puede definirse como toda la variedad o diversidad de formas de vida que habita el Planeta. Por su relevancia para el ser humano, la conservación, manejo y uso de la biodiversidad es un tema que en la actualidad demanda la atención de un creciente número de científicos. Así, es común que los resultados de estos estudios estén asociados a una lista de los organismos estudiados. Sin embargo, no puede referirse a los seres vivos como si solamente fuesen listas de organismos, ya que las especies no están aisladas, sino que están ligadas y moldeadas por las interacciones ecológicas. Por lo tanto, para conservar, manejar y usar la biodiversidad, deben conservarse también las interacciones entre las especies (Thompson, 1996).

Las interacciones interespecíficas son la diferencia básica entre los seres vivos en una comunidad en condiciones naturales y los organismos en un jardín botánico o en un parque zoológico. Éstos han sido extraídos del medio que los moldeó, cortando de tajo sus relaciones intra e interespecíficas y las posibilidades evolutivas que los otros miembros de su especie enfrentan en condiciones naturales. Así como las especies han evolucionado y se han multiplicado, lo han hecho igual las interacciones que enlazan sus historias de vida y dan forma a su evolución futura (Thompson, 1982). A pesar de que las interacciones son generalmente más efímeras y menos tangibles que las especies, no dejan de ser ciertamente un producto evolutivo, al igual que el hueso, la quitina o una pared celular. Por lo tanto, el estudio de los orígenes y evolución de las interacciones entre especies es tan crucial para el entendimiento de la evolución de la vida, como lo es el estudio de los orígenes y la evolución de las especies (Thompson, 1994).

Existen una serie de teorías y desacuerdos para explicar la alta diversidad encontrada en las regiones tropicales (Huston, 1994). Sin embargo, existe poco desacuerdo con respecto al importante papel de las interacciones interespecíficas (Rico-Gray, 2001) y, entre ellas, las interacciones planta-animal son claramente de alto interés (Howe y Westley, 1988). Por ejemplo, las interacciones planta-herbívoro son de gran importancia pues los herbívoros, como consumidores de productividad primaria, 
son capaces de ejercer poderosas influencias sobre la estructura y funcionamiento de los ecosistemas. Muchos de los problemas económicos más importantes en ecosistemas tropicales perturbados están relacionados con la infestación de cultivos por herbívoros (Farnworth y Golley, 1975). El propósito de este trabajo es presentar la importancia que éstas interacciones tienen en la agricultura y en la conservación y manejo de la biodiversidad.

\section{IMPORTANCIA DEL ESTUDIO DE LAS INTERACCIONES PARA LA AGRICULTURA}

Los agroecosistemas son áreas donde los procesos físicos, químicos y biológicos son manipulados por el ser humano para la producción de alimento y/o fibra (incluyendo forestal y tejidos). La agricultura engloba a un conjunto de prácticas y manipulaciones requeridas para producir los productos deseados para el uso humano. La estructura y la función del ecosistema natural han sido modificadas dramáticamente en la mayor parte del mundo por la agricultura. La trasformación es un proceso histórico y actual, afectando a los procesos ecológicos a nivel de la población, comunidad y ecosistema; además de ser una de las más poderosas fuerzas antropogénicas modificadoras del paisaje. Cuando un bosque o pradera es aclarado y arado, el cambio más notable es la alteración dramática en la estructura de la vegetación, que trae como consecuencia un profundo impacto sobre la distribución de los organismos, así como de las interacciones (Stinner y Stinner, 1989). Aunado a esto, se suceden cambios en las propiedades del suelo y en las condiciones microclimáticas, que también tienen influencia sobre la distribución de los organismos y de sus interacciones.

Cuando un bosque es talado, arado y repoblado con un cultivo, la composición de especies cambia fuertemente. Las especies dominantes cambian de especies locales, que evolucionaron recíprocamente con otras especies, a especies que en muchos casos evolucionaron en diferentes regiones geográficas y en diferentes tipos de ecosistemas. Las especies cultivadas traen generalmente asociadas plagas, usualmente insectos. Adicionalmente, la introducción de cultivos exóticos puede traer también a especies asociadas de plantas. Además de los efectos directos sobre la composición de especies asociados al reemplazamiento de las especies dominantes, también se presentan efectos indirectos. Estos pueden tener mayor impacto sobre la composición total de especies y diversidad de los ecosistemas agrícolas, que los efectos directos (Stinner y Stinner, 1989). Los cambios en la arquitectura de las plantas y en la diversidad del paisaje como resultado de las prácticas agrícolas, tendrán fuerte influencia en la presencia y distribución de muchas especies de animales (Risch, Andow y Altieri, 1983; Forman y Gordon, 1986). Lawton y Schroder (1977) demostraron que la densidad de insectos y la riqueza de especies se depaupera al transformar un bosque y pasar a una zona de hierbas y anuales. El arado y cultivo de un terreno cambian apreciablemente el ambiente físico del suelo y estimulan la mineralización de nutrientes, de una forma relativamente inmóvil a una móvil. Este proceso afecta posteriormente el contenido de nutrientes en los tejidos de las plantas (Stinner et al., 1984), que a su vez puede influenciar los niveles de herbivoría (Scriber, 1984). Aunado a esto, el arado y el cultivo influencían significativamente a las poblaciones de depredadores que habitan en el suelo, que también, a su vez, pueden influenciar a las poblaciones de herbívoros (House y Stinner, 1983; Brust, Stinner y McCartney, 1986).

La agricultura afecta la cantidad y tipos de interacciones entre los organismos, ya que generalmente reduce la composición de especies y la diversidad. El repertorio de interacciones que ocurren entre las plantas y animales en los ecosistemas agrícolas es generalmente mucho más restringido que el de un ecosistema natural (Stinner y Stinner, 1989). El arado y la aplicación de insecticidas disminuyen los niveles tróficos superiores, tales como los depredadores y parásitos que ayudan a regular las poblaciones de herbívoros (Price, 1984; Brust, Stinner y McCartney, 1985). La aplicación de insecticidas también puede afectar el número de polinizadores, particularmente los insectos, en cultivos que requieren de su participación para la producción de frutos (Bentley, 1983). A pesar de que hay poca evidencia directa de como afectan los procesos agrícolas a las proporciones relativas de los dife- 
rentes tipos de interacciones (p.ej., antagonismo, mutualismo), se ha hipotetizado que al pasar de un ecosistema natural a uno agrícola deben de incrementarse las interaciones antagónicas (p.ej., cultivo-malezas, planta-patógeno, planta-herbívoro) (Crossley et al., 1984; Stinner y Stinner, 1989). Es en estas interacciones antagónicas donde el ser humano gasta grandes cantidades de energía (mecánica y química) tratando de controlarlas. Para tratar de no disminuir drásticamente la biodiversidad, debe considerarse a la labranza asociada a un proceso de conservación, a los cultivos múltiples, a nuevas especies de cultivo, a la genética recombinante y a la biotecnología (Stinner y Stinner, 1989).

\section{IMPORTANCIA DEL ESTUDIO DE LAS INTERACCIONES PARA LA CONSERVA- CIÓN DE LA BIODIVERSIDAD}

Se pueden identificar cuatro características estrictamente bióticas en interacciones interespecíficas, probablemente comunes a muchos ecosistemas terrestres, que son de importancia clave para entender, manejar y conservar una parte importante de la biodiversidad neotropical: mosaico químico y tramas alimenticias coevolucionadas, enlaces móviles, mutualistas clave y mosaico de hormigas (Gilbert, 1980) (Figura 1).

Mosaico químico y tramas alimenticias coevolucionadas.- Los bosques neotropicales son mosaicos excesivamente complejos con respecto a la variedad interespecífica de compuestos secundarios

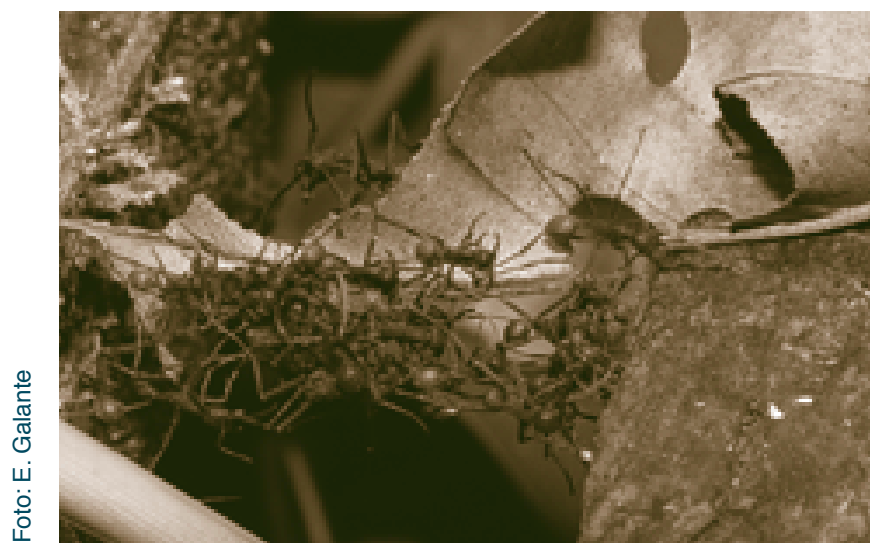

Figura 1. Hormiga legionaria. Bosque neotropical. (p.ej., alcaloides, terpenos), químicos que se sugiere tienen una función defensiva en las plantas. Consecuentemente, la mayoría de los insectos herbívoros han evolucionado especializaciones sensoriales y digestivas que restringen su dieta a una fracción relativamente pequeña y químicamente similar de las especies de plantas disponibles. Una característica importante del mosaico químico es el hecho que las plantas del mismo género o familia compartan generalmente muchos compuestos, así como a aquellos insectos especializados para manejar tales compuestos. Una gran fracción de los insectos herbívoros, junto con sus propios parasitoides, están a su vez organizados dentro de muchas cadenas alimenticias separadas. Estas cadenas ocurren de manera muy cercana, pero están basadas en diferentes compartimentos de la producción primaria, química y taxonómicamente delimitados. El referirse a tales sistemas como cadenas alimenticias coevolucionadas (Gilbert, 1977), refleja que los patrones del mosaico químico han sido y continuarán siendo generados por un proceso evolutivo entre las plantas y sus parásitos y herbívoros (Gilbert, 1980). Por otra parte, cabe la posibilidad de que los insectos se diversificaron contra un mosaico existente de químicos en las plantas y no han tenido impacto selectivo en el mosaico químico (Gilbert, 1980). Indistintamente a cómo evolucionó la subestructura de un ecosistema, el hecho importante para la biología de la conservación es que la mayor parte de la diversidad total de especies en un bosque neotropical se encuentra en muchas cadenas alimenticias paralelas, hospedero-restringidas, que son similares en organización trófica pero diferentes taxonómicamente. Si entendemos como se mantiene la diversidad dentro de una muestra representativa de los cientos de tales subsistemas, podríamos encontrar reglas generales para manejar el sinnúmero de especies de las cuales sabemos poco o nada.

Enlaces móviles.- Se conoce como enlaces móviles a los animales que son un factor significativo para la persistencia de una variedad de especies de plantas las cuales, a su vez, soportan tramas alimenticias separadas (Gilbert, 1980). La mayoría de las plantas de los bosques tropicales, además de ser 
raras y en muchos casos presentar patrones dispersos de distribución, requieren polinización cruzada (Bawa, 1974), y dependen de los colibríes (Feinsinger, 1983), las abejas (Delgado y Sousa, 1977), las mariposas nocturnas y los murciélagos (Heithaus, Fleming \& Opler, 1975) para su polinización. Asímismo, se requiere de una dispersión eficiente de semillas para la colonización de sitios adecuados, la germinación y el establecimiento de las plántulas (Ortíz-Pulido y Rico-Gray 2000). Las aves y los murciélagos son los más importantes dispersores de semillas en los neotrópicos (Estrada y Fleming, 1986). Las hormigas también pueden ser importantes para el movimiento de las semillas (Cuautle, et al., 2005), algunas patrullan y defienden a una variedad de plantas productoras de néctar (RicoGray y Thien, 1989; Cuautle y Rico-Gray, 2003; Rico-Gray et al., 2004) y la protección por hormigas puede ser importante en el establecimiento de las plántulas, así como en la reproducción de la planta adulta. Debe evaluarse y manejarse la diversidad de los enlaces móviles y éstos deben ser el centro de atención de la investigación ecológica dentro de la conservación biológica.

Mutualistas clave.- Se conoce como mutualistas clave a aquellos organismos, típicamente plantas, que proveen soporte crítico a complejos grandes de enlaces móviles. La pérdida de un mutualista clave causaría, con cierto desfasamiento en el tiempo, la pérdida de enlaces móviles $y$, posteriormente, como consequencia del resquebrajamiento en la reproducción y la dispersión, la pérdida de plantas dependientes del enlace (Serio-Silva y Rico-Gray, 2003). Finalmente, la diversidad de insectos hospederoespecífico debe declinar con la reducción en la diversidad de la comunidad de hospederos (Futuyma, 1973).

Mosaico de hormigas.- Las relaciones entre hormigas y plantas pueden ser de muchos tipos, por ejemplo (Keeler, 1989) (Figura 2):

\section{A. Antagonismos:}

1. Depredación de semillas

2. Hormigas cortadoras de hojas

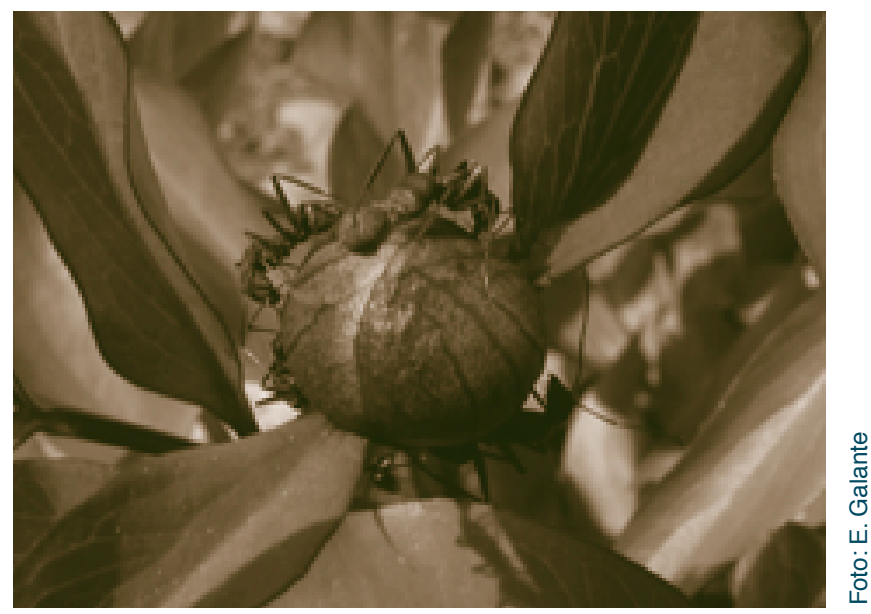

Figura 2. Mutualismo hormiga del género Camponotus-planta

\section{B. Mutualismos:}

3. Nectarios extraflorales

4. Corpúsculos alimenticios y sitios de anidación

5. Plantas alimentadas por hormigas

6. Jardines de hormigas

7. Dispersión de semillas (primaria y secundaria)

8. Polinización

9. Sistemas homiga-Hemiptera/Lepidoptera-planta

\section{Interacciones indirectas:}

\section{Modificación del suelo}

Se sabe de la existencia de un mosaico tridimensional de hormigas que resulta del no solapamiento de los territorios de las pocas especies de hormigas dominantes en la comunidad. Con cada hormiga dominante coexisten conjuntos diferentes de hormigas subdominantes, de tal manera que el mosaico está conformado por parches de comunidades de hormigas. Una consecuencia importante del mosaico de hormigas para la agricultura y la conservación, es el hecho que la inmunidad de una planta a ciertas enfermedades o a organismos defoliadores es una función de su posición en el mosaico de hormigas (p.ej., Gilbert, 1980). El conocimiento del mosaico de hormigas puede ser importante para el manejo de pequeñas reservas neotropicales. Si es reducido el número de individuos reproductivos de una especie de mutualista 
clave, la defoliación de parte o toda la población por hormigas cortadoras de hojas (Atta spp.) o monos aulladores (Alouatta spp.), puede ser suficiente para eliminar inmediatamente a importantes enlaces móviles, con la subsecuente pérdida de especies dependientes de éstos (Gilbert, 1980). Así como en África se manipulan los mosaicos de hormigas para incrementar la producción de cacao (Majer, 1976), también podría ser manipulado el mosaico en reservas de poco tamaño para asegurar la floración y fructificación de los mutualistas clave.

La estructura de los bosques neotropicales está altamente organizada y determinada por las interacciones planta-animal. El sistema consiste de muchas cadenas o tramas alimenticias paralelas, estructuralmente similares pero taxonómicamente diferentes, basadas en grupos particulares de plantas. Los mutualismos juegan un papel crucial en el mantenimiento de la biodiversidad del sistema. Los enlaces móviles son animales requeridos por muchas plantas para su reproducción y dispersión. Los mutualistas clave son plantas que soportan a los enlaces móviles e indirectamente soportan las cadenas alimenticias; éstas, a su vez, dependen de los enlaces móviles para mantener toda o parte de su riqueza de especies. Finalmente, porque las hormigas controlan el grado de herbivoría sobre muchas plantas, la existencia de un mosaico de diferentes hormigas territoriales y dominantes, crea una forma sutil, pero importante, de mosaico. Estas interacciones ocurren en un sistema de parches de microhábitats, definidos por el tamaño del disturbio y el estado sucesional. Muchos organismos clave en el sistema están restringidos a un microhábitat, mientras que otros, especialmente los enlaces móviles, dependen de la disponibilidad constante de varios microhábitats.

\section{REFERENCIAS}

BAWA, K. S. 1974. Breeding systems of tree species of a lowland tropical community. Evolution 28:85-92.

BENTLEY, B. L. 1983. Nectaries in agriculture, with an emphasis on the tropics. In: B. L. Bentley y T. S. Elias (eds.), The Biology of Nectaries. Columbia University Press, New York. Pp. 204-222.
BRUST, G. E., B. R. STINNER y D. A. MCCARTNEY. 1985. Tillage and soil insecticide effects on predators - black cutworm (Lepidoptera: Noctuidae) interactions in corn agroecosystems. J. Econ. Entomol. 78: 1389-1392.

BRUST, G. E., B. R. STINNER y D. A. MCCARTNEY. 1986. Predator activity and predation in corn agroecosystems. Environ. Entomol. 15:1017-1021.

CROSSELY, D. A., G. J. HOUSE, R. M. SNIDER y B. R. STINNER. 1984. Positive interactions in agroecosystems. In: R. Lowrence, B. R. Stinner y G. J. House (eds.), Agricultural Ecosystems. John Wiley and Sons, New York. Pp. 73-82.

CUAUTLE, M. y V. RICO-GRAY. 2003. The effect of wasps and ants on the reproductive success of the extrafloral nectaried plant Turnera ulmifolia (Turneraceae). Functional Ecology 17: 417-423.

CUAUTLE, M., V. RICO-GRAY y C. DÍAZCASTELAZO. 2005. Effects of ant behaviour and extrafloral nectaries presence on seed dispersal of the neotropical myrmecochore Turnera ulmifolia L. (Turneraceae), in a sand dune matorral. Biological Journal of the Linnean Society, en prensa.

DELGADO, A. y M. SOUSA. 1977. Biología floral del género Cassia en la región de Los Tuxtlas, Veracruz. Bol. Soc. Bot. Méx. 37:5-52.

ESTRADA, A. y T. H. FLEMING (eds.). 1986. Frugivores and Seed Dispersal. Dr W. Junk Publishers, Dorcrecht.

FARNWORTH, E. G. y F. B. GOLLEY (eds.). 1975. Fragile Ecosystems: evaluation of research and applications in the Neotropics. Springer-Verlag, New York.

FEINSINGER, P. 1983. Coevolution and pollination. In: D. J. Futuyma y M. Slatkin (eds.), Coevolution. Sinauer Associates Press, Sunderland, MA. Pp. 282-310.

FORMAN, R. T. T. y M. Gordon. 1986. Landscape Ecology. John Wiley and Sons, New York.

FUTUYMA, D. J. 1973. Community structure and stability in constant environments. Am. Nat. 107:443-446. 
GILBERT, L. E. 1977. The role of insect-plant coevolution in the organization of ecosystems. In: V. Labyrie (ed.), Comportment des Insectes et Milieu Trophique.C.N.R.S., Paris. Pp. 399-413.

GILBERT, L. E. 1980. Food web organization and the conservation of neotropical diversity. In: M. E. Soulé y B. A. Wilcox (eds.), Conservation Biology. Sinauer Associates Press, Sunderland, MA. Pp. 11-33.

HEITHAUS, E. R., T. H. FLEMING y P. A. OPLER. 1975. Foraging patterns an resource utilization of seven species of bats in a seasonal tropical forest. Ecology 56:841-854.

HOUSE, G. J. y B. R. STINNER. 1983. Arthropods in non-tillage soybean agroecosystems: community composition and ecosystem interactions. Environ. Manag. 7:23-28.

HOWE, H. F. y L. C. WESTLEY. 1988. Ecological Relationships of Plants and Animals. Oxford University Press, Oxford.

HUSTON, M. A. 1994. Biological Diversity. Cambridge University Press, Cambridge.

KEELER, K. H. 1989. Ant-plant interactions. In: W. G. Abrahamson (ed.), Plant-Animal Interactions. McGraw-Hill Book Company, New York. Pp. 207-242.

LAWTON, J. H. y D. SCHRODER. 1977. Effects of plant type, size of geographical range and taxonomic isolation on number of insect species associated with British plants. Nature 265:137-140.

MAJER, J. D. 1976. The maintenance of the ant mosaic in Ghana cocoa farms. J. Appl. Ecol. 13:123-144.

ORTIZ-PULIDO, R. y V. RICO-GRAY. 2000. The effect of spatio-temporal variation in understanding the fruit crop size hypothesis. Oikos 91:523-527.

PRICE, P. W. 1984. Insect Ecology. John Wiley and Sons, New York.

RICO-GRAY, V. 2001. Interspecific interaction. In: Encyplopedia of Life Sciences. Macmillan Press (www.els.net), London. Pp. 1-6.

RICO-GRAY, V. y L. B. THIEN. 1989. Effect of different ant species on reproductive fit- ness of Schomburgkia tibicinis (Orchidaceae). Oecologia 81:487-489.

RICO-GRAY, V., P.S. OLIVEIRA, V. PARRATABLA, M. CUAUTLE y C. DÍAZ-CASTELAZO. 2004. Ant-plant interactions: their seasonal variation and effects on plant fitness. In: M.L. Martínez y N. Psuty (eds.), Coastal Sand Dunes: Ecology and Restoration, Ecological Studies 171. Springer-Verlag, Berlin. Pp. 221-239.

RISCH, S. J., D. ANDOW y M. H. ALTIERI. 1983. Agroecosystem diversities and pest control: data, tentative conclusions, and new research directions. Environ. Entomol. 12:625-629.

SCRIBER, J. M. 1984. Host plant suitability. In: W. J. Bell y R. T. Corde (eds.), Chemical Ecology of Insects. Suncer and CMA: Sinauer Associates, Sunderland, MA. Pp.160-202.

SERIO-SILVA, J. C. y V. RICO-GRAY. 2003. The role of howler monkeys (Alouatta palliata mexicana) as seed dispersal agents of strangler figs (Urostigma) in disturbed and preserved habitats in south Veracruz, México. In: L. K. Marsh (ed.), Primates in Fragments: ecology and conservation. Klewer Academic/Plenum Publishers, New York. Pp. 267-281.

STINNER, B. R. y D. S. STINNER. 1989. Plant-animal interactions in agricultural ecosystems. In: W. G. Abrahamson (ed.), Plant-Animal Interactions. McGraw-Hill Book Company, New York. Pp. 355-393.

STINNER, B. R., D. A. CROSSELY, E. P. ODUM y R. L. TODD. 1984. Nutrient budgets and internal cycling of $\mathrm{N}, \mathrm{P}, \mathrm{K}, \mathrm{Ca}$ and $\mathrm{Mg}$ in conventional tillage, no-tillage and old-field ecosystems on the Georgia piedmont. Ecology 65:354-369.

THOMPSON, J. N. 1982. Interaction and Coevolution. John Wiley and Sons, New York.

THOMPSON, J. N. 1994. The Coevolutionary Process. University of Chicago Press, Chicago.

THOMPSON, J. N. 1996. Evolutionary ecology and the conservation of biodiversity. Trends Ecol. Evol. 11:300-303. 